\title{
A FUNCTIONAL EQUATION FROM PROBABILITY THEORY
}

\author{
JOHN A. BAKER
}

(Communicated by J. Marshall Ash)

ABstract. The functional equation

$$
f(x)=\prod_{j=1}^{N}\left[f\left(\beta_{j} x\right)\right]^{\gamma_{j}}
$$

has been used by Laha and Lukacs (Aequationes Math. 16 (1977), 259-274) to characterize normal distributions. The aim of the present paper is to study (1) under somewhat different assumptions than those assumed by Laha and Lukacs by using techniques which, in the author's opinion, are simpler than those employed by the afore-mentioned authors. We will prove, for example, that if $0<\beta_{j}<1$ and $\gamma_{j}>0$ for $1 \leq j \leq N, \sum_{j=1}^{N} \beta_{j}^{k} \gamma_{j}=1$, where $k$ is a natural number, $f: \mathbb{R} \rightarrow[0,+\infty)$, (1) holds for $x \in \mathbb{R}$ and $f^{(k)}(0)$ exists then either $f \equiv 0$ or there exists a real constant $c$ such that $f(x)=\exp \left(c x^{k}\right)$ for all $x \in \mathbb{R}$.

\section{INTRODUCTION}

In [3, p. 237] we find the following theorem attributed to Vincze [6].

Theorem A. Suppose $\sigma, a, b>0, a^{2}+b^{2}=1, \varphi \in \mathbb{R} \rightarrow \mathbb{C}$,

$$
\varphi(x)=\sigma \sqrt{2 \pi} \varphi(a x) \varphi(b x) \quad \text { for all } x \in \mathbb{R},
$$

$\varphi^{\prime \prime}(0)$ exists, and $\int_{\mathbf{R}} \varphi(x) d x=1$. Then

$$
\varphi(x)=(1 / \sigma \sqrt{2 \pi}) \exp \left(-x^{2} / \sigma^{2}\right) \text { for all } x \in \mathbb{R} .
$$

Notice that if we let $f(x)=\sigma \sqrt{2 \pi} \varphi(x)$ for $x \in \mathbb{R}$ then (2) is equivalent to (1) with $N=2, \beta_{1}=a, \beta_{2}=b, \gamma_{1}=\gamma_{2}=1$ and, in this instance, $\beta_{1}^{2} \gamma_{1}+\beta_{2}^{2} \gamma_{2}=1$.

Suppose $F: \mathbb{R} \rightarrow \mathbb{R}, F$ is nondecreasing and continuous on the right, $\lim _{t \rightarrow-\infty} F(t)=0$, and $\lim _{t \rightarrow+\infty} F(t)=1$. If

$$
f(x)=\int_{-\infty}^{\infty} e^{i x t} d F(t) \quad \text { for } x \in \mathbb{R},
$$

then $f$ is called the characteristic function of the distribution function $F$ (see [5]). Thus a characteristic function $f$ is, by definition, the Fourier-Stieltjes

Received by the editors June 23, 1992 and, in revised form, October 9, 1992.

1991 Mathematics Subject Classification. Primary 39B12, 60E10; Secondary 39B22.

Key words and phrases. Functional equation, probability. 
transform of a distribution function and therefore, as is well known, $f$ is continuous, $f(0)=1$ and $f$ is positive definite in the sense of Bochner.

In [5], Laha and Lukacs proved

Theorem B. If $0<\beta_{j}<l$ and $\gamma_{j}>0$ for $1 \leq j \leq N, \sum_{j=1}^{N} \beta_{j}^{2} \gamma_{j}=1, f$ is $a$ characteristic function, and (1) holds for all $x \in \mathbb{R}$ then $f$ is the characteristic function of a normal distribution with zero mean.

The conclusion is equivalent to asserting that $f=\varphi$ where $\varphi$ is defined by (3) for some $\sigma>0$.

Theorem $\mathrm{A}$ is proved in [3] by using general results concerning certain classes of functional equations. Theorem B is deduced in [5] from a part of the theory of characteristic functions involving the Fourier transform. Our results will be based on the comparatively simple propositions of the next section.

Throughout this paper $N$ is a given natural number and $\beta_{1}, \ldots, \beta_{N}$, $\gamma_{1}, \ldots, \gamma_{N}$ are given real numbers such that $0<\beta_{j}<1$ and $\gamma_{j}>0$ for $1 \leq j \leq N$. We also let

$$
p_{j}=-\ln \beta_{j}>0 \quad \text { for } 1 \leq j \leq N .
$$

If we let $\rho(s)=\sum_{j=1}^{N} \beta_{j}^{s} \gamma_{j}$ for $s \in \mathbb{R}$ then $\rho$ is continuous, strictly decreasing, $\lim _{s \rightarrow+\infty} \rho(s)=0$, and $\lim _{s \rightarrow-\infty} \rho(s)=+\infty$. Hence there is a unique real number $k$ such that

$$
\sum_{j=1}^{N} \beta_{j}^{k} \gamma_{j}=1
$$

Let $\mu_{j}=\beta_{j}^{k} \gamma_{j}>0$ for $1 \leq j \leq N$ so that $\sum_{j=1}^{N} \mu_{j}=1$.

Notice that $k=2$ in both of the above theorems. We will be mainly concerned with cases in which $k$ is a natural number and $f$ satisfies some regularity condition at (or near) zero.

We denote the natural numbers by $\mathbb{N}$, the integers by $\mathbb{Z}$, the real numbers by $\mathbb{R}$, and the complex numbers by $\mathbb{C}$.

Although we will eventually consider cases of (1) for functions $f$ from $\mathbb{R}$ to $\mathbb{R}$ (or $\mathbb{C}$ or even a Banach algebra), we will begin by concentrating on functions $f$ from $(0,+\infty)$ to $(0,+\infty)$; the main ideas of our proofs are more easily understood in this setting.

\section{TWO BASIC PROPOSITIONS}

Proposition 1. Suppose $f:(0,+\infty) \rightarrow(0,+\infty)$. Then (1) holds for all $x>0$ if and only if there exists a function $\varphi: \mathbb{R} \rightarrow \mathbb{R}$ such that

$$
\begin{aligned}
& f(x)=\exp \left[x^{k} \varphi(\ln x)\right] \quad \text { for all } x>0, \\
& \text { i.e., } \varphi(t)=e^{-k t} \ln f\left(e^{t}\right) \quad \text { for all } t \in \mathbb{R},
\end{aligned}
$$

and

$$
\varphi(t)=\sum_{j=1}^{N} \mu_{j} \varphi\left(t-p_{j}\right) \quad \text { for all } t \in \mathbb{R}
$$


Proof. Suppose (1) holds for all $x>0$ and let $\psi(t)=\ln f\left(e^{t}\right)$ for $t \in \mathbb{R}$. Then, by $(1), \psi(t)=\ln \left\{\prod_{j=1}^{N}\left[f\left(\beta_{j} e^{t}\right)\right]^{\gamma_{j}}\right\}=\sum_{j=1}^{N} \gamma_{j} \ln f\left(e^{-p_{j}} e^{t}\right)$, i.e.,

$$
\psi(t)=\sum_{j=1}^{N} \gamma_{j} \psi\left(t-p_{j}\right) \quad \text { for all } t \in \mathbb{R} .
$$

For $s \in \mathbb{R}$ let $\psi_{s}(t)=e^{-s t} \psi(t)$ for $t \in \mathbb{R}$. By $(6)$ and $(*)$,

$$
\begin{aligned}
\psi_{s}(t) & =e^{-s t} \sum_{j=1}^{N} \gamma_{j} \psi\left(t-p_{j}\right)=\sum_{j=1}^{N} \gamma_{j} e^{-s p_{j}} e^{-s\left(t-p_{j}\right)} \psi\left(t-p_{j}\right) \\
& =\sum_{j=1}^{N} \beta_{j}^{s} \gamma_{j} \psi_{s}\left(t-p_{j}\right) \quad \text { for } s, t \in \mathbb{R} .
\end{aligned}
$$

If we let $\varphi=\psi_{k}$ then we have (5) and $\psi(t)=e^{k t} \psi_{k}(t)=\left(e^{t}\right)^{k} \varphi(t)$ for all $t \in \mathbb{R}$.

Thus $f(x)=\exp [\psi(\ln x)]=\exp \left[x^{k} \varphi(\ln x)\right]$ for all $x>0$, i.e., (4) holds. The converse involves only a simple computation.

The next result is a generalization of the simple fact that if $\varphi: \mathbb{R} \rightarrow \mathbb{R}, \varphi$ is periodic, and $\lim _{t \rightarrow-\infty} \varphi(t)$ exists, then $\varphi$ is constant.

Proposition 2. Suppose $\varphi: \mathbb{R} \rightarrow \mathbb{R}$ (or any normal linear space), (5) holds, and $\lim _{t \rightarrow-\infty} \varphi(t)$ exists. Then $\varphi$ is constant.

Proof. Suppose $\varphi(t) \rightarrow l \in \mathbb{R}$ as $t \rightarrow-\infty$. Given $\varepsilon>0$, choose $a \in \mathbb{R}$ such that $|\varphi(t)-l|<\varepsilon$ whenever $t<a$. Let $p=\min \left\{p_{1}, \ldots, p_{N}\right\}>0$. If $t<a+p$ then $t-p_{j}<a$ for $1 \leq j \leq N$ so that $\left|\varphi\left(t-p_{j}\right)-l\right|<\varepsilon$. Hence if $t<a+p$ then, by (\#) and the definition of $\mu_{j}$,

$$
|\varphi(t)-l|=\left|\sum_{j=1}^{N} \mu_{j}\left\{\varphi\left(t-p_{j}\right)-l\right\}\right|<\sum_{j=1}^{N} \mu_{j} \varepsilon=\varepsilon .
$$

By induction, $|\varphi(t)-l|<\varepsilon$ provided $t<a+n p$ for some $n \in \mathbb{N}$. It follows that

$$
|\varphi(t)-l|<\varepsilon \quad \text { for all } t \in \mathbb{R} .
$$

Since this is so for every $\varepsilon>0$ we must conclude that $\varphi(t)=l$ for all $t \in \mathbb{R}$.

Proposition 3. Suppose $f:(0,+\infty) \rightarrow[0,+\infty)$, (1) holds for all $x>0$, $\sum_{j=1}^{N} \gamma_{j} \geq 1$ (equivalently, $k \geq 0$ ), and $\lim _{x \rightarrow 0+} f(x)$ exists. Then either $f \equiv 0$ or $f(x)>0$ for all $x \geq 0$. Moreover assuming $f \not \equiv 0$, if $k=0$ then $f$ is constant and if $k>0$ then $\lim _{x \rightarrow 0+} f(x)=1$.

Proof. Let $c=\lim _{x \rightarrow 0+} f(x)$. By (1), $c=c^{\gamma}$ where $\gamma=\sum_{j=1}^{N} \gamma_{j} \geq 1$.

Suppose first that $c=0$. Let $0<\varepsilon<1$ and choose $\delta>0$ such that $0 \leq f(x)<\varepsilon$ whenever $0<x<\delta$. Let $\beta=\max \left\{\beta_{1}, \beta_{2}, \ldots, \beta_{N}\right\}$ so that $0<\beta<1$. If $0<x<\delta / \beta$ then $0<\beta_{j} x \leq \beta x<\delta$ for $1 \leq j \leq N$ and hence

$$
0 \leq f(x)=\prod_{j=1}^{N}\left[f\left(\beta_{j} x\right)\right]^{\gamma_{j}}<\varepsilon^{\gamma} \leq \varepsilon
$$


since $0<\varepsilon<1$ and $\gamma=\sum_{j=1}^{N} \gamma_{j} \geq 1$. That is $0 \leq f(x)<\varepsilon$ whenever $0<x<\delta / \beta$. By induction, for any natural number $n, 0 \leq f(x)<\varepsilon$ whenever $0<x<\delta / \beta^{n}$ from which it follows that $0 \leq f(x)<\varepsilon$ for all $x>0$. Since this is so for any $\varepsilon \in(0,1)$ we conclude that $f \equiv 0$ if $c=0$.

Suppose next that $c>0$ and $\gamma=\sum_{j=1}^{N} \gamma_{j}=1$ (i.e., $k=0$ ). Since $\lim _{x \rightarrow 0+} f(x)>0$, it follows from (1) that $f(x)>0$ for all $x>0$. Let $\psi(t)=\ln f\left(e^{t}\right)$ for $t \in \mathbb{R}$ so that, by (6),

$$
\psi(t)=\sum_{j=1}^{N} \gamma_{j} \psi\left(t-p_{j}\right) \quad \text { for all } t \in \mathbb{R} \text { and } \lim _{t \rightarrow-\infty} \psi(t)=\ln c=: d \text {. }
$$

By Proposition 2, $\psi$ is constant and hence $f$ is constant.

Suppose finally that $c>0$ and $\sum_{j=1}^{N} \gamma_{j}>1$, i.e., $k>0$. To complete the proof it suffices to show that $f(x)>0$ for all $x>0$ and $c=1$. But $c=c^{\gamma}$, $c>0$, and $\gamma=\sum_{j=1}^{N} \gamma_{j}>1$, so $c=1$. Hence $f$ is positive in $(0, \delta)$ for some $\delta>0$ and it follows from (1) that $f$ must be positive on $(0,+\infty)$.

Corollary. Suppose that $f:[0,+\infty) \rightarrow[0,+\infty)($ or $f: \mathbb{R} \rightarrow[0,+\infty)),(1)$ holds for all $x \geq 0$, (resp. all $x \in \mathbb{R}), k=0$, and $f$ is continuous at 0 . Then $f$ is constant.

Proof. Proposition 3 implies that $f$ is constant on $(0,+\infty)$ and hence $f$ is constant on $[0,+\infty)$ so the proof is complete if the domain of $f$ is $[0,+\infty)$.

Suppose $f: \mathbb{R} \rightarrow[0,+\infty)$. Let $g(x)=f(-x)$ for all $x>0$. Then

$$
g(x)=f(-x)=\prod_{j=1}^{N}\left[f\left(\beta_{j}(-x)\right)\right]^{\gamma_{j}}=\prod_{j=1}^{N}\left[g\left(\beta_{j} x\right)\right]^{\gamma_{j}}
$$

for all $x>0$ and $\lim _{x \rightarrow 0+} g(x)=\lim _{x \rightarrow 0} f(x)$. Hence by Proposition $3, g$ is constant on $(0,+\infty)$. Thus $f$ is constant on $(-\infty, 0)$. Using the continuity of $f$ at 0 once again we conclude the $f$ is constant on $\mathbb{R}$.

\section{THE MAIN RESULTS}

Theorem 1. Suppose $f: \mathbb{R} \rightarrow[0,+\infty)$, (1) holds for all $x \in \mathbb{R}, k \in \mathbb{N}$, and $f^{(k)}(0)$ exists. Then either $f \equiv 0$ or there exists $c \in \mathbb{R}$ such that

$$
f(x)=\exp \left[c x^{k}\right] \quad \text { for all } x \in \mathbb{R} \text {. }
$$

Proof. Since $f^{\prime}(0)$ exists, $f$ is continuous at 0 . By Proposition 3 (applied to the restriction of $f$ to $(0,+\infty))$, either $f(x)=0$ for all $x \geq 0$ or $f(0)=1$ and $f(x)>0$ for all $x>0$. Assume the latter.

Let $F(x)=\ln f(x)$ for $x \in \mathbb{R}$. Choose $\varphi: \mathbb{R} \rightarrow \mathbb{R}$ such that (4) and (5) hold.

Then $f(x)=x^{k} \varphi(\ln x)$ for all $x>0, F(0)=0$, and $F^{\prime}(0)=f^{\prime}(0) / f(0)=$ $f^{\prime}(0)$.

Suppose $k=1$. Let

$$
c=F^{\prime}(0)=\lim _{x \rightarrow 0+} \frac{F(x)-F(0)}{x}=\lim _{x \rightarrow 0+} \varphi(\ln x)=\lim _{t \rightarrow-\infty} \varphi(t) .
$$

By Proposition 2, $\varphi(t)=c$ for all $t \in \mathbb{R}$. Hence $F(x)=c x$ for all $x>0$ and thus $f(x)=\exp [c x]$ for all $x>0$. The continuity of $f$ at 0 thus implies that 
$f(x)=\exp [c x]$ for all $x \geq 0$. Let $g(x)=f(-x)$ for $x \in \mathbb{R}$. Then

$$
g(x)=f(-x)=\prod_{j=1}^{N} f\left(\beta_{j}(-x)\right)^{\gamma_{j}}=\prod_{j=1}^{N} g\left(\beta_{j} x\right)^{\gamma_{j}}
$$

for all $x \in \mathbb{R}$ and $g^{\prime}(0)=-f^{\prime}(0)=-c$. Hence $g(x)=e^{-c x}$ for all $x>0$. That is $f(x)=e^{c x}$ for all $x<0$. Thus our assertion is true in the case $k=1$.

Now suppose that $k \geq 2$. By assumption, there exists $\Delta>0$ such that $f^{(k-1)}(x)$ is defined for all $x \in(-\Delta, \Delta)$. Hence $\varphi^{(k-1)}(t)$ is defined for all $t \in(-\infty, \ln \Delta)$ and hence, by (5), for all $t \in \mathbb{R}$. It then follows from (4) that $f^{(k-1)}(x)$ and $F^{(k-1)}(x)$ are defined for all $x>0$. Now for all $x>0$

$$
F(x)=x^{k} \varphi(\ln x)
$$

and

$$
F^{\prime}(x)=k x^{k-1} \varphi(\ln x)+x^{k} x^{-1} \varphi^{\prime}(\ln x)
$$

or

$$
F^{\prime}(x)=x^{k-1}\left\{\varphi^{\prime}(\ln x)+k \varphi(\ln x)\right\} .
$$

For $\alpha \in \mathbb{R}$ let $D+\alpha$ denote the linear differential operator defined by

$$
([D+\alpha] \psi)(t)=\psi^{\prime}(t)+\alpha \psi(t) \quad \text { for all } t \in \mathbb{R}
$$

whenever $\psi: \mathbb{R} \rightarrow \mathbb{R}$ is differentiable. Then

$$
F^{\prime}(x)=x^{k-1}([D+k] \varphi)(\ln x) \quad \text { for all } x>0 .
$$

Similarly, if $k \geq 3$ we have, for all $x>0$,

$$
\begin{aligned}
F^{\prime \prime}(x) & =x^{k-2}([D+k-1][D+k] \varphi)(\ln x), \\
& \vdots \\
F^{(k-1)}(x) & =x([D-2] \cdots[D+k] \varphi)(\ln x) .
\end{aligned}
$$

Let $\psi=[D+2] \cdots[D+k] \varphi$. Then

$$
\psi=\varphi^{(k-1)}+a_{k-2} \varphi^{k-2}+\cdots+a_{1} \varphi^{\prime}+a_{0} \varphi \quad \text { where } a_{0}, a_{1}, \ldots, a_{k-2} \in \mathbb{R}
$$

are such that, for all $z \in \mathbb{C}$,

$$
z^{k-1}+a_{k-2} z^{k-2}+\cdots+a_{1} z+a_{0}=(z+2) \cdots(z+k) .
$$

Notice that $a_{0}=k$ ! It then follows from (5) that

$$
\psi(t)=\sum_{j=1}^{N} \mu_{j} \psi\left(t-p_{j}\right) \quad \text { for all } t \in \mathbb{R} .
$$

We also know that

$$
F^{(k-1)}(x)=x \psi(\ln x) \quad \text { for all } x>0 .
$$

Now $F(x)=\ln f(x)=\sum_{j=1}^{N} \gamma_{j} \ln f\left(\beta_{j} x\right)=\sum_{j=1}^{N} \gamma_{j} F\left(\beta_{j} x\right)$ for all $x \in \mathbb{R}$ so that

$$
F^{\prime}(x)=\sum_{j=1}^{N} \beta_{j} \gamma_{j} F^{\prime}\left(\beta_{j} x\right) \quad \text { for all } x \in \mathbb{R} .
$$


Hence $F^{\prime}(0)=\left\{\sum_{j=1}^{N} \beta_{j} \gamma_{j}\right\} F^{\prime}(0)$. But $\sum_{j=1}^{N} \beta_{j} \gamma_{j}>\sum_{j=1}^{N} \beta_{j}^{k} \gamma_{1}=1$ since $k \geq 2$ so $F^{\prime}(0)=0$. If $k \geq 3$ then

$$
F^{\prime \prime}(x)=\sum_{j=1}^{N} \beta_{j}^{2} \gamma_{j} F^{\prime \prime}\left(\beta_{j} x\right) \quad \text { for all } x \in \mathbb{R}
$$

so that $F^{\prime \prime}(0)=0$ since $\sum_{j=1}^{N} \beta_{j}^{2} \gamma_{j}>\sum_{j=1}^{N} \beta_{j}^{k} \gamma_{j}=1$. Continuing this argument we find that $0=F^{(m)}(0)$ for $m=1,2, \ldots, k-1$.

But $F^{(k)}(0)$ exists since $f^{(k)}(0)$ exists and we know that $F^{(k-1)}(0)=0$. Hence, by $(8)$,

$$
F^{(k)}(0)=\lim _{x \rightarrow 0+} \frac{F^{(k-1)}(x)-F^{(k-1)}(0)}{x}=\lim _{x \rightarrow 0+} \psi(\ln x) .
$$

By (7) and Proposition 2, $\psi$ is constant, say $\psi(t)=\lambda \in \mathbb{R}$ for all $t \in \mathbb{R}$. That is, for all $t \in \mathbb{R}$,

$$
\varphi^{(k-1)}(t)+a_{k-2} \varphi^{(k-2)}(t)+\cdots+a_{1} \varphi^{1}(t)+(k !) \varphi(t)=\lambda .
$$

Hence there exist real constants $c_{2}, \ldots, c_{k}$ such that

$$
\varphi(t)=c_{2} e^{-2 t}+\cdots+c_{k} e^{-k t}+(\lambda / k !) \quad \text { for all } t \in \mathbb{R} .
$$

Thus

$$
\begin{aligned}
F(x) & =x^{k} \varphi(\ln x)=x^{k}\left[\frac{c_{2}}{x^{2}}+\cdots+\frac{c_{k}}{x^{k}}+\left(\frac{\lambda}{k !}\right)\right] \\
& =\left(\frac{\lambda}{k !}\right) x^{k}+c_{k}+\cdots+c_{2} x^{k-2} \quad \text { for all } x>0 .
\end{aligned}
$$

Since $0=F^{(m)}(0)$ for $m=1,2, \ldots, k-1$, we have $c_{2}=\cdots=c_{k}=0$. Thus, if $c=\lambda / k$ !, then $F(x)=c x^{k}$ for all $x>0$ and hence, for all $x>0$,

$$
f(x)=\exp \left[c x^{k}\right] \text {. }
$$

Arguing as we did in the case $k=1$ we find that (9) holds for all real $x$.

\section{GeNERALIZATIONS}

If $S$ is a (multiplicative) semigroup and $\gamma_{1}, \ldots, \gamma_{N} \in \mathbb{N}$ then one can make sense of $(1)$ for functions $f$ from $\mathbb{R}$ (or $(0,+\infty)$ ) into $S$.

Suppose that $A$ is a (real or complex) commutative Banach algebra with identity 1 . For $a \in A$ define

$$
\exp (a)=\sum_{n=0}^{\infty}(1 / n !) a^{n}
$$

As is well known, $\exp$ is a $C^{\infty}$ map of $A$ into $A, \exp (0)=1, \exp ^{\prime}(0)$ is the identity map of $A$ onto itself, and $\exp (a+b)=(\exp a)(\exp b)$ whenever $a$, $b \in A$. By the Inverse Function Theorem (see, e.g., [7, p. 172]) there exists a neighbourhood $U$ of 0 in $A$ and a neighbourhood $V$ of 1 in $A$ such that the restriction of exp to $U$ is a bijection of $U$ onto $V$ whose inverse, call it $L$, is $C^{\infty}$.

Suppose $\gamma_{j} \in \mathbb{N}$ for $1 \leq j \leq N$ and let $\gamma=\sum_{j=1}^{N} \gamma_{j}$. Choose a neighbourhood $W$ of 1 in $A$ such that $w_{1} w_{2} \cdots w_{\gamma} \in V$ whenever $w_{1}, w_{2}, \ldots, w_{\gamma} \in$ $W$. It follows that $L\left(w_{1} \cdots w_{\gamma}\right)=\sum_{j=1}^{\gamma} L\left(w_{j}\right)$ for all $w_{1}, \ldots, w_{\gamma} \in W$.

With this machinery we can generalize some of the above results as follows. 
Theorem 2. With the above assumptions, suppose $f: \mathbb{R} \rightarrow A$ satisfies (1) for all $x \in \mathbb{R}$. If $k \in \mathbb{N}, f^{(k)}=0$ exists, and $f(0)$ is invertible then there exist $a, c \in A$ such that

$$
a=a^{\gamma} \quad \text { and } \quad f(x)=a \exp \left[x^{k} c\right] \quad \text { for all } x \in \mathbb{R} .
$$

Sketch of proof. First note that if $a=f(0)$ then, by (1), $a=a^{\gamma}$. If we let $f_{1}(x)=a^{-1} f(x)$ for $x \in \mathbb{R}$, then $f_{1}(0)=1, f_{1}(x)=a^{-1} \prod_{j=1}^{N}\left[f\left(\beta_{j} x\right)\right]^{\gamma_{j}}=$ $\prod_{j=1}^{N}\left[a^{-1} f\left(\beta_{j} x\right)\right]^{\gamma_{j}}$, and $f^{(k)}(0)$ exists. Thus we may assume that $f(0)=1$. The existence of $f_{1}^{(k)}(0)$ implies the continuity of $f$ at 0 . This, in turn, in light of (1), implies that $f(x)$ is invertible for all $x>0$. Moreover there exists $\Delta>0$ such that $f(x) \in W$ whenever $-\Delta<x<\Delta$. Let $\varphi(t)=e^{-k t} L\left[f\left(e^{t}\right)\right]$ for $-\infty<t<\ln \Delta$. Then $\varphi(t)=\sum_{j=1}^{N} \beta_{j}^{k} \gamma_{j} \varphi\left(t-p_{j}\right)$ for all $t \in(-\infty, \ln \Delta)$. The proof can be completed by slightly modifying the proof of Theorem 1 .

Remarks 1. A result similar to Theorem 2 can also be obtained without assuming that $\gamma_{1}, \ldots, \gamma_{N}$ are all in $\mathbb{N}$. Assume that $\gamma_{j}>0$ for $1 \leq j \leq N$, and $\sum_{j=1}^{N} \gamma_{j}=\gamma<M$ for some $M \in \mathbb{N}$. Let $W^{\prime}=\{w \in V: \mu L(w) \in U$ for all $\mu \in(0, M)\}$ and define

$$
w^{\mu}=\exp [\mu L(w)] \quad \text { for } w \in W^{\prime} \text { and } \mu \in(0, M) .
$$

Suppose $\Delta>0, f:(-\Delta, \Delta) \rightarrow W^{\prime},(1)$ holds for all $x \in(-\Delta, \Delta)$, and $f(0)=$ 1 . Then by slightly modifying the above ideas one can prove that if $k \in \mathbb{N}$ and $f^{(k)}(0)$ exists then there exists $c \in A$ such that $f(x)=\exp \left[x^{k} c\right]$ for all $x \in(-\Delta, \Delta)$.

2. In Theorem 2 it is not necessary to assume that $A$ is commutative. Instead one could assume that $f(x) f(y)=f(y) f(x)$ for all $x, y \in \mathbb{R}$ and replace $A$ by the closed subalgebra of $A$ generated by $\{f(x): x \in \mathbb{R}\} \cup\{1\}$. For if this subalgebra is denoted by $B$ then $f$ maps $\mathbb{R}$ into $B$ and $B$ is commutative.

3. Our results were obtained by reducing (1) to (6). Equation (6) has also been studied under a variety of conditions different from those we have assumed (see, for example, $[1,2,4]$ ).

\section{REFERENCES}

1. John A. Baker, Functional equations, tempered distributions and Fourier transforms, Trans. Amer. Math. Soc. 315 (1989), 57-68.

2. W. Jarczyk, A recurrent method of solving iterative functional equations, Uniwersytet Slaski, Katowice, 1991.

3. M. Kuczma, B. Choczewski, and R. Ger, Iterative functional equations, Cambridge Univ. Press, London and New York, 1990.

4. M. Laczkovich, Non-negative measurable solutions of a difference equation, J. London Math. Soc. (2) 34 (1986), 139-147.

5. R. G. Laha and E. Lukacs, On a functional equation which occurs in a characterization problem, Aequationes Math. 16 (1977), 259-274.

6. E. Vincze, Bemerkung zur Charakterisierung der Gauss'schen Fehlergesetzes, Magyar Tud. Acad. Mat. Kutató Int. Kösl. 7 (1962), 357-61.

7. E. Zeidler, Nonlinear functional analysis and its applications. I, Springer-Verlag, New York, Berlin, Heidelberg, and Tokyo, 1986.

Department of Pure Mathematics, University of Waterloo, Waterloo, Ontario, Canada N2L 3G1 